\title{
A novel homozygous mutation in the WNK1/HSN2 gene causing hereditary sensory neuropathy type 2
}

\author{
Anna Potulska-Chromik\#1, Dagmara Kabzińska\#2, Marta Lipowska1 ${ }^{1}$ Anna Kostera-Pruszczyk ${ }^{1 凶}$ \\ and Andrzej Kochański2,3
}

1Department of Neurology, Medical University of Warsaw, Poland; 2Neuromuscular Unit, Mossakowski Medical Research Centre, Polish Academy of Sciences, Warsaw, Poland; 3Faculty of Biology and Environmental Sciences, Cardinal Stefan Wyszyński, University in Warsaw, Warsaw, Poland

\begin{abstract}
Hereditary sensory and autonomic neuropathy type 2 is a rare disorder caused by recessive mutations in the WNK1/HSN2 gene located on chromosome 12p13.33. Phenotype of the patients is characterized by severe sensory loss affecting all sensory modalities. We report a novel homozygous Lys179fsX182 (HSN2); Lys965fsX968 (WNK1/HSN2) mutation causing an early childhood onset hereditary sensory and autonomic neuropathy type 2, with acromutilations in upper and lower limbs, and autonomic dysfunction. To the best of our knowledge this is the first genetically proven case of hereditary sensory and autonomic neuropathy type 2 originating from East Europe.
\end{abstract}

Key words: sensory neuropathy, hereditary, autonomic, mutation, sympathetic skin response

Received: 30 May, 2012; revised: 23 July, 2012; accepted: 13 August, 2012; available on-line: 21 August, 2012

\section{INTRODUCTION}

Hereditary sensory neuropathies are a genetically and clinically heterogeneous group of disorders, primarily affecting the peripheral sensory system. Most patients present with sensory loss and insensitivity to pain, that result in chronic ulcerations or even extensive infections leading to amputations of fingers or toes.

Some childhood onset cases were attributed to mutations of the with-no-lysine $(\mathrm{K})-1$ (WNK1) gene, transmitted in an autosomal recessive mode. Large intronic deletions within the WNK1 gene were first reported in families with pseudohypoaldosteronism type II (OMIM 145260).

Later studies demonstrated that mutations of HSN2, a nervous system-specific exon of the-WNK1, cause hereditary sensory neuropathy type II (HSAN2A; OMIM 201300). Since the first report 11 different mutations of the WNK1/HSN2 gene were reported (Human Mutations Database). Most of the HSAN2 cases were observed in consanguineous families (autosomal recessive trait of inheritance); nevertheless, in some patients only one mutation in the WNK1/HSN2 gene was detected.

For the molecular genetic purposes HSAN2 families have been identified in a genetically isolated Newfoundland population in the early 1990s. This isolate population is characterized by a multiplied founder effect for numerous disorders resulting from the immigration of the Protestant and Roman Catholic settlers to Newfoundland in the XIX century (Rahman et al., 2003). The genetic linkage analysis studies were performed in two HSAN2 families. A large F1family was previously char- acterized at the clinical level by Ogryzlo and coworkers in 1946 (Ogryzlo, 1946).

In 2004 Lafreniere et coworkers mapped HSAN2 to the $12 \mathrm{p} 13.33$ region. As a result of an exhaustive search they identified an unknown open reading frame (ORF) located within intron 8 of the WNK1 gene. In this ORF, encompassing one exon-long gene, they identified three homozygous mutations in the patients originating from Newfoundland (c. 594del A), Nova Scotia (c. 918-919 ins A) and a nonsense mutation in a French Canadian patient (c. 943 C>T) (Lafreniere et al., 2004).

The ORF of the gene responsible for the HSAN2 phenotype was designated as the HSN2 gene (Lafreniere, MacDonald, Dube, MacFarlane, O’Driscoll, Brais, Meilleur, Brinkman, Dadivas, Pape, Platon, Radomski, Risler, Thompson, Guerra-Escobio, Davar, Breakefield, Pimstone, Green, Pryse-Phillips, Goldberg, Younghusband, Hayden, Sherrington, Rouleau, \& Samuels, 2004). Later on, the HSN2 gene has been shown not to be an autonomous gene since its transcript was detected in a longerWNK1/HSN2 isoform expressed in the neural tissue.

Especially high expression of the WNK1/HSN2 isoforms was found in sensory components of the peripheral nervous system (Shekarabi et al., 2008). Thus, mutations in the WNK1 gene should be reported in the traditional nomenclature (HSN2) and in a new manner (WNK1/HSN2).

In the subsequent studies mutations in the WNK1/ HSN2 gene were identified also beyond the French Canadian and Newfoundland populations. The WNK1/ HSN2 gene mutations were found in Lebanese, Austrian, Italian, Belgian and Japanese HSAN2 affected patients (Auer-Grumbach et al., 2006; Takagi et al., 2006). To the best of our knowledge no mutations in the WNK1/ HSN2 gene have been reported it the Middle and East European population.

We report a novel mutation in the WNK1/HSN2 gene resulting in the HSAN2 phenotype in our index case.

\section{CASE REPORT}

Our proband is a 12.5-years old girl, born at term after uncomplicated pregnancy. Her family history was

\footnotetext{
e-mail: akostera@wum.edu.pl
}

"contributed equally to this work

Abbreviations: EMG, electromyography; HSN2, a nervous systemspecific exon of the WNK1; HSNA2, hereditary sensory and autonomic neuropathy type 2; ORF, open reading frame, OMIM, Online Mendelian Inheritance; in Man, PubMed, database of references and abstracts on life sciences and biomedical topics; SSR, sympathetic skin responses; WNK1, with-no-lysine(K)-1 gene 


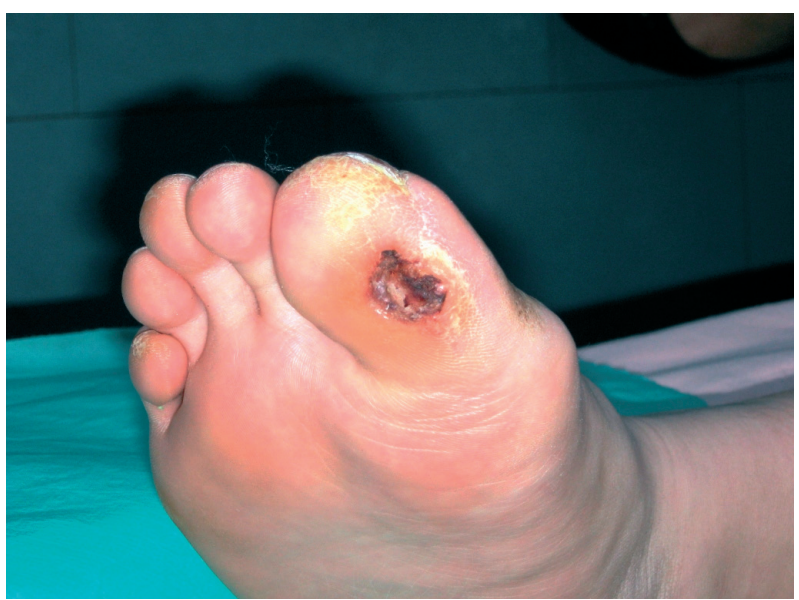

Figure 1. Trophic changes on the right toe of the proband.

not relevant, she has two older and one younger healthy sisters. Her parents are not related, however, their families have been living in the same small village for several generations.

Her developmental milestones were normal. She had some swallowing difficulties noted in the first year of life. Since her early childhood the parents noticed that she did not cry when hurt. She was prone to burns and unnoticed injuries. When she was 5 , she had a painless fracture of one of her metatarsal bones. At that time a neuropathy with insensitivity to pain was suspected. Since the age of 8 she had recurrent fever episodes, not related to infection.

She was first seen at our department a year ago. She was in a good condition. Examination revealed sensory loss in her upper extremities up to elbows, and in the lower extremities up to her knees. All sensory modalities were affected. She had areflexia in her lower extremities.

No muscle weakness or atrophy was present. She had ulceration and swelling of her right foot. The nails of fingers and toes were dysplastic. Her blood pressure was normal; there were no clinical symptoms of dysautonomia. Her mental development was also normal. A year later her sensory loss progressed. She
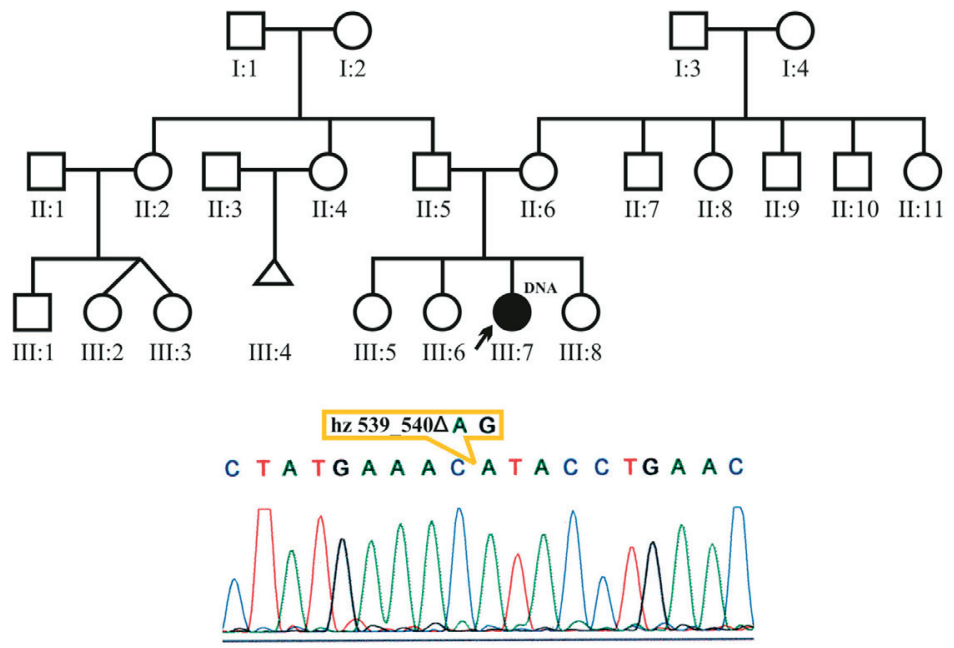

Figure 2. Pedigree chart of three-generational family with HSAN II (upper panel). The proband III:7 is indicated with an arrow. A homozygous two nucleotide AG

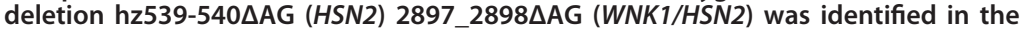
proband III:7 in the HSN2/WNK1 gene (lower panel). continued to have trophic changes on her right foot (Fig. 1).

Electrophysiological studies revealed absent sensory nerve action potentials. Motor nerve conduction studies and concentric needle EMG results were within normal limits. Auditory sympathetic skin responses (SSR) were absent. SSR with electrical stimulation elicited responses with prolonged latencies to her palms, while no responses were recordable in the soles. Measurement of the R-R interval in her electrocardiogram gave a normal result.

Neurological exam and EMG of both her parents were normal.

DNA analysis methods and results. The blood sample was taken from the proband (III:7). The family members participating in this study gave their informed consent. The study was approved by the local ethics committee at the Warsaw Medical University; No. of approval $120 / 2008$.

The whole genomic DNA was isolated from white blood cells by the desalting procedure. In the proband (III:7), first, a duplication and deletion of the peripheral myelin protein 22 gene (PMP22) were excluded in the real-time PCR (Q-PCR) approach (Aarskog \& Vedeler, 2000).

A sequence of the $W N K 1$ gene corresponding to the HSN2 region was amplified in the PCR reaction with designed 5 pairs of primers. The WNK/HSN2 region was directly sequenced using the BigDyeTM Terminator Version 1.1 Ready Reaction Cycle Sequencing kit on the ABI 3730/xl genetic analyzer (Applied Biosystem).

Two systems of mutation's nomenclature have been used. For the traditional/historical HSN2 nomenclature the reference sequences i.e. NM_213655.2 (mRNA) and NC_000012.11 (genomic sequence) were applied. For the new WNK1/HSN2 gene nomenclature two reference sequences NM_213655.3 (mRNA) and NG_007984.2 (genomic sequence) were used.

The PMP22 gene dosage analysis revealed a normal dosage of the PMP22 gene.

Direct sequencing of the WNK1/HSN2 gene revealed a homozygous two -nucleotide deletion 539_540 $\mathrm{AG}$ (NM-213655.2-HSN2)/ 2897_2898AAG (NM-213655.3WNK1/HSN2), leading to a frame-shift mutation resulting in a premature stop codon (by conceptual translation) at position 182 of the HSN2 protein (Lys179fsX182) or Lys965fsX968 (WNK1/HSN2) (Fig. 2).

\section{DISCUSSION}

To the best of our knowledge we report the first WNK1/HSN2 mutation in a HSAN2 affected patient originating from Eastern European population. An earlier report described two Polish patients with recessive hereditary sensory neuropathy, but no molecular studies were conducted in them (Jedrzejowska \& Milczarek, 1976).

Given the rules of conceptual translation, this new homozygous Lys179fsX182/Lys965fsX968 mutation in the WNK1/HSN2 gene results in a nonfunctional protein.

All mutations of the WNK1/HSN2 gene reported to date have been shown to result in the frame shift or codon 
stop and to be loss-of-function mutations (Coen et al., 2006; Rotthier et al., 2009; Shekarabi et al., 2008).

There is no possibility to make reliable phenotypegenotype correlations for WNK1/HSN2 mutations since the vast majority of them represent private mutations occurring in single families or even in single patients.

Only the S307fsX319 mutation found in a French Canadian was detected in 13 families due to a founder effect in an isolate population (Roddier et al., 2005).

Interestingly, all the reported French Canadian patients living in the southern part of the Quebec province in Canada presented a relatively homogeneous phenotype. All of them had first HSAN symptoms in the first decade of life. All of them developed foot, toe, finger or hand infections in the first decade of life. Necrosis was observed in the patients starting from the beginning of the second decade. The patients in the second, third or fourth decade had undergone leg amputations, which indicates slowly progressive nature of HSAN2 (Roddier et al., 2005). Our patient presented with congenital sensory loss leading to multiple injuries and a painless bone fracture. Although she did not have prominent autonomic disturbances, such as hyperhidrosis, urinary incontinence or slow pupillary reaction to light, her sympathetic skin response results were grossly abnormal. Abnormalities in SSR in hereditary sensory neuropathies are attributed to anhidrosis or hyperkeratotic skin, and are a constant feature of HSAN IV. In contrast, SSR are preserved in HSAN III (Hilz et al., 1999; Hilz \& Dutsch, 2006). Interestingly, our patient also reported several episodes of fever, a symptom not frequent in HSAN II (Rotthier et al., 2009).

There is no doubt that the Lys179fsX182 (HSN2); Lys965fsX968 (WNK1/HSN2) variant detected in our study is a pathogenic mutation causative for the HSAN2 phenotype observed in the proband. We decided not to perform sequencing analysis of the whole WNK1 gene due to the prior detection of a homozygous, deleterious Lys179fsX182 mutation in the HSN2region. The whole sequence of $W N K 1$ should be analyzed only in the HSAN2 affected patients, in whom no mutations have been found in the HSN2 region.

Our case report expands the list of mutations of WNK1/HSN2 causing hereditary sensory and autonomic neuropathy type II. It also proves the presence of this rare disease in East-Europeans.

\section{Acknowledgements}

We thank the HSAN family for active participation in this study. We are also grateful to Izabela Podstawka, MSc, and Mrs. Jadwiga Kędzierska for skillful technical assistance.

This study was supported by the Polish Ministry of Science and Higher Education (grant no. N N402 2763 36 to $\mathrm{AK})$.

\section{REFERENCES}

Aarskog NK, Vedeler CA (2000) Real-time quantitative polymerase chain reaction $\mathrm{A}$ new method that detects both the peripheral myelin protein 22 duplication in Charcot-Marie-Tooth type $1 \mathrm{~A}$ disease and the peripheral myelin protein 22 deletion in hereditary neuropathy with liability to pressure palsies. Hum Genet 107: 494-498.

Auer-Grumbach M, Mauko B, Auer-Grumbach P, Pieber TR (2006) Molecular genetics of hereditary sensory neuropathies. Neuromolecular Med 8: 147-158.

Coen K, Pareyson D, Auer-Grumbach M, Buyse G, Goemans N, Claeys KG, Verpoorten N, Laura M, Scaioli V, Salmhofer W, Pieber TR, Nelis E, De JP, Timmerman V (2006) Novel mutations in the HSN2 gene causing hereditary sensory and autonomic neuropathy type II. Neurology 66: 748-751.

Hilz MJ, Dutsch M (2006) Quantitative studies of autonomic function. Muscle Nerve 33: 6-20.

Hilz MJ, Stemper B, Axelrod FB (1999) Sympathetic skin response differentiates hereditary sensory autonomic neuropathies III and IV. Neurology 52: 1652-1657.

Jedrzejowska H, Milczarek H (1976) Recessive hereditary sensory neuropathy. J Neurol Sci 29: 371-387.

Lafreniere RG, MacDonald ML, Dube MP, MacFarlane J, O'Driscoll M, Brais B, Meilleur S, Brinkman RR, Dadivas O, Pape T, Platon C, Radomski C, Risler J, Thompson J, Guerra-Escobio AM, Davar G, Breakefield XO, Pimstone SN, Green R, Pryse-Phillips W, Goldberg YP, Younghusband HB, Hayden MR, Sherrington R, Rouleau GA, Samuels ME (2004) Identification of a novel gene (HSN2) causing hereditary sensory and autonomic neuropathy type II through the Study of Canadian Genetic Isolates. Am J Hum Genet 74: $1064-1073$.

Ogryzlo MA (1946) A familial peripheral neuropathy of unknown etiology resembling Morvan's disease. Can Med Assoc J 54: 547-553.

Rahman P, Jones A, Curtis J, Bartlett S, Peddle L, Fernandez BA, Freimer NB (2003) The Newfoundland population: a unique resource for genetic investigation of complex diseases. Hum Mol Genet 12 (Spec No 2): R167-R172.

Roddier K, Thomas T, Marleau G, Gagnon AM, Dicaire MJ, St-Denis A, Gosselin I, Sarrazin AM, Larbrisseau A, Lambert M, Vanasse M, Gaudet D, Rouleau GA, Brais B (2005) Two mutations in the HSN2 gene explain the high prevalence of HSAN2 in French Canadians. Neurology 64: 1762-1767.

Rotthier A, Baets J, De VE, Jacobs A, Auer-Grumbach M, Levy N, Bonello-Palot N, Kilic SS, Weis J, Nascimento A, Swinkels M, Kruyt MC, Jordanova A, De JP, Timmerman V (2009) Genes for hereditary sensory and autonomic neuropathies: a genotype-phenotype correlation. Brain 132: 2699-2711.

Shekarabi M, Girard N, Riviere JB, Dion P, Houle M, Toulouse A, Lafreniere RG, Vercauteren F, Hince P, Laganiere J, Rochefort D, Faivre L, Samuels M, Rouleau GA (2008) Mutations in the nervous system - specific HSN2 exon of WNK1 cause hereditary sensory neuropathy type II. J Clin Invest 118: 2496-2505.

Takagi M, Ozawa T, Hara K, Naruse S, Ishihara T, Shimbo J, Igarashi S, Tanaka K, Onodera O, Nishizawa M (2006) New HSN2 mutation in Japanese patient with hereditary sensory and autonomic neuropathy type 2. Neurology 66: 1251-1252. 
